\title{
Combined Efficacy of Adrenal Vein Sampling and Imaging Findings in Predicting Clinical Outcomes Following Unilateral Adrenalectomy for Primary Aldosteronism
}

\author{
Rajiv N. Srinivasa ${ }^{1}$ Matthew E. Anderson ${ }^{1} \quad$ Alan P. Dackiw ${ }^{2} \quad$ Anil K. Pillai ${ }^{1} \quad$ Clayton K. Trimmer $^{1}$ \\ Sanjeeva P. Kalva ${ }^{1}$ Stephen P. Reis ${ }^{3}$
}

${ }^{1}$ Division of Interventional Radiology, Department of Radiology, University of Texas Southwestern Medical Center, Dallas,

Texas, United States

2 Division of Endocrine Surgery, Department of Surgery, University of

Texas Southwestern Medical Center, Dallas, Texas, United States

${ }^{3}$ Department of Radiology, Columbia University Medical Center,

New York, New York, United States

J Clin Interv Radiol ISVIR 2017;1:71-76.

\author{
Address for correspondence Rajiv N. Srinivasa, MD, Department of \\ Radiology, University of Texas Southwestern Medical Center, 5323 \\ Harry Hines Blvd, Dallas, TX 75390, United States \\ (e-mail: Rajiv.Srinivasa@PHHS.org).
}

\begin{abstract}
Keywords

- adrenal vein sampling

- primary aldosteronism

- adrenalectomy

The objective of this study was to investigate the combined efficacy of adrenal vein sampling (AVS) and imaging findings in predicting successful clinical outcomes following unilateral adrenalectomy for primary aldosteronism (PA). A retrospective chart review of 137 patients who underwent AVS between 2009 and 2014 at two hospitals in a single academic institution was performed. Preprocedure demographic, imaging, medication, and laboratory values were reviewed. In general, patients were considered for adrenalectomy when lateralization was suggested on AVS. Clinical outcomes such as improved blood pressure control and preserved renal function after adrenalectomy were correlated with preprocedure variables. AVS was technically successful in 120 out of 137 patients. Lateralization was seen in 64 patients and 48 out of 64 patients underwent adrenalectomy. Out of 48,43 patients had an adrenal nodule on preoperative imaging, while 5 did not. 28 patients showed improvement in blood pressure after adrenalectomy, all of which had a nodule on imaging. Of the 28 patients, 22 also showed preservation of renal function. None of the remaining 5 (out of 48) patients who demonstrated lateralization on AVS and had no nodule on imaging showed clinical improvement following adrenalectomy. The presence of an adrenal nodule on preoperative imaging was also associated with improved blood pressure control $(p=0.022)$ and preserved renal function $(p=0.048)$ following adrenalectomy. Improved blood pressure control and preserved renal function in patients with PA who underwent adrenalectomy following lateralization on AVS are associated with the identification of an adrenal nodule on preoperative imaging.
\end{abstract}

\section{Introduction}

Adrenal vein sampling (AVS) has been described as a method for localizing hypersecreting adrenal disease in patients with primary aldosteronism (PA). ${ }^{1}$ PA most often results from either an adrenal adenoma or adrenal hyperplasia and is associated with excess aldosterone secretion. The most common presenting symptom in these patients is drug- 
resistant hypertension. The diagnosis is often delayed and these patients are seen in specialty hypertension clinics and are frequently treated with multiple antihypertensives. Cardiovascular and cerebrovascular sequelae of hypertension are a major cause of morbidity and mortality in patients with PA. ${ }^{2,3}$ In addition, end-organ damage to the kidneys frequently results in chronic renal insufficiency, which leads to further morbidity and mortality. ${ }^{4-6}$

Patients with suspected PA undergo laboratory testing to evaluate for elevated peripheral blood aldosterone and suppressed renin levels. Further confirmatory testing may be performed with saline suppression or salt loading. Nearly all patients with evidence of excess aldosterone secretion have some form of cross-sectional imaging of the abdomen performed to evaluate the adrenal glands for the presence of a nodule. In addition, AVS is utilized to determine if one adrenal gland is acting autonomously to hypersecrete aldosterone relative to the contralateral gland. Adrenal vein blood samples which demonstrate elevated secretion of aldosterone by one gland are said to have lateralized. In general, if lateralization of aldosterone secretion is identified after AVS, the patient may undergo adrenalectomy with the intention of improving blood pressure control on fewer medications. ${ }^{7}$

The overall rate of cure measured by improvement in blood pressure control after unilateral adrenalectomy is quite variable and has been reported in the literature ranging from 33 to $82 \% .^{7,8}$ Given the unacceptably high number of adrenalectomy procedures performed without benefit, it is important to reconsider the factors that are associated with clinical improvement and cure of hypertension in different populations of PA patients. The primary objective of this study is to determine factors during the work-up of PA patients that can predict who is likely to have improved blood pressure control and stable renal function after adrenalectomy. As a secondary objective, we evaluate differences in treatment outcomes among ethnic subgroups.

\section{Materials and Methods}

\section{Study Population}

This retrospective review was Health Insurance Portability and Accountability Act (HIPAA) compliant and received approval from our Institutional Review Board. Informed consent was waived. The radiology Picture Archiving and Communication System database was reviewed for all AVS procedures performed at either of the two hospitals at a large academic institution between May 2009 and April 2014. A total of 137 procedures were identified with technical success in 120 (88\%), which were utilized in further analysis. Technical failure was due to inability to sample the right adrenal vein in 10 cases, the left adrenal vein in 6 cases, and both adrenal veins in 1 case. The study cohort which included only technically successful cases consisted of 68 males and 52 females with an average age of 52.0 years (range, 21-79 years).

\section{Data Collection}

A review of the electronic health records including demographic information of each patient who underwent AVS was performed. The number of antihypertensive medications each patient was taking before and after adrenalectomy as recorded at the most recent follow-up visit (mean 6.3 months) was collected. Laboratory measures including peripheral vein aldosterone and renin levels prior to AVS were recorded. Measures obtained from AVS included peripheral vein, inferior vena cava (IVC), and right and left adrenal vein cortisol and aldosterone levels. Values are reported as mean \pm standard deviation, unless otherwise stated. All patients received a cosyntropin infusion $(0.25 \mathrm{mg}$ in $250 \mathrm{~mL}$ of $5 \%$ dextrose in water infused at $50 \mathrm{~mL}$ per hour) prior to AVS. Successful sampling was confirmed by venographic appearance and by rapid cortisol assay. An adrenal vein cortisol level more than three times the IVC level was used as the selectivity index cutoff to determine technically successful sampling prior to the patient being discharged. The aldosterone and cortisol measures were then used to calculate the lateralization index (LI) for each patient, which is defined as the higher aldosterone-to-cortisol (AC) ratio divided by the lower AC ratio on the contralateral side. In general, an $\mathrm{LI} \geq 4.0$ was considered positive for lateralization and served as a pre-requisite for consideration of adrenalectomy. In addition, estimated glomerular filtration rate (eGFR) was calculated using the Modification of Diet in Renal Disease formula. Baseline serum creatinine measurements obtained prior to AVS and at the most recent follow-up visit (mean, 8.4 months) were collected to determine change in eGFR. Available abdominal computed tomography (CT) and/ or magnetic resonance (MR) imaging studies performed prior to adrenalectomy were also reviewed for all patients. Imaging included both adrenal and nonadrenal protocols CT and MR. Images were categorized with regard to the presence or absence of an adrenal nodule on imaging and were reviewed by a board-certified radiologist. Finally, surgical pathology was reviewed for all patients who underwent adrenalectomy.

\section{Statistical Analysis}

The number of antihypertensive medications a given patient was taking was used as a surrogate measure of the clinical severity of PA. The primary measure of clinical success in our study was achieving a decrease in the number of antihypertensive medications by at least one following adrenalectomy. This measure of clinical success has been utilized in previous studies by Wang et al and Pirvu et al. ${ }^{9,10}$ A complete cure was deemed when the number was reduced to zero after adrenalectomy. A secondary outcome utilized in this study was end-organ damage to the kidneys. It has been reported that a decline in eGFR of $30 \%$ over 2 years is associated with an increased risk of end-stage renal disease (ESRD) and mortality. ${ }^{11}$ In this study, we utilized a drop in eGFR of more than $30 \%$ over our shorter timespan averaging 8.4 months from baseline prior to AVS to follow-up postadrenalectomy to indicate end-organ damage. Fisher's exact test was performed to identify factors associated with clinical improvement as measured by these two outcomes, with $p=0.05$ signifying statistical significance. Differences in treatment outcomes were also investigated for Caucasian, 
African American, and Hispanic subgroups. All lateralization indices were calculated utilizing Excel (Microsoft) and all statistical analyses were performed in SPSS (version 22, SPSS, IBM). Not all clinical measures were available for every individual.

\section{Results}

A review of all AVS cases performed at our institution revealed technical success in 120 out of 137 cases (88\%).

-Fig. 1 shows a flowchart of all AVS patients with their clinical course and outcomes. Of the technically successful cases, 64 patients had an $\mathrm{LI} \geq 4.0$ and of those, 48 underwent adrenalectomy. The remaining 16 patients were subsequently determined to be poor operative candidates or were lost to follow-up. A total of 28 patients (58\%) who underwent adrenalectomy demonstrated improved blood pressure control with $3(6 \%)$ taken off all antihypertensives. All these 28 patients (100\%) with clinical improvement had a discrete nodule seen on preoperative imaging on the side of lateralization. The $\mathrm{LI}$ range for the 28 patients with treatment success and the 20 patients without success showed no statistically significant difference ( $p=0.467)$.

There were 56 patients who underwent technically successful AVS but did not lateralize ( $\mathrm{LI}<4.0$ ), 21 of these patients had a nodule on imaging. Despite not lateralizing on AVS, six patients in this group still underwent adrenalectomy at the discretion of the surgeon and in correspondence with the patient's desires, while the remaining patients were treated medically. All six patients who underwent adrenalectomy in this group had a discrete nodule seen on preoperative imaging, but only one patient showed clinical improvement postadrenalectomy. Notably, this patient had both an adrenal nodule and adrenal gland thickening seen on imaging. The remaining 50 patients who did not lateralize on AVS were treated medically, with 7 (14\%) demonstrating clinical improvement over the timespan of the study. Four of these patients had a nodule on imaging, two had adrenal gland thickening, and one had normal appearing adrenal glands. - Table 1 shows a summary of the characteristics of all patients who underwent adrenalectomy.

Surgical pathology revealed an adenoma in $80 \%(n=43)$ and adrenal hyperplasia in the remaining $20 \%(n=11)$ who underwent adrenalectomy. In the group that lateralized on AVS and had clinical improvement after surgery, 25 (89\%) had an adrenal adenoma on surgical pathology, while the remaining 3 (11\%) showed adrenal hyperplasia. In the group that lateralized but did not have clinical improvement after surgery, 16 (80\%) had adrenal adenomas on surgical pathology, while 4 (20\%) had adrenal hyperplasia. Of the six patients who did not lateralize on AVS but still underwent adrenalectomy, surgical pathology revealed two with adenomas and four with adrenal hyperplasia. Of note, one patient in this group who did have clinical improvement had adrenal hyperplasia at surgical pathology. These results are summarized in $\mathbf{- T a b l e ~} \mathbf{2}$.

Out of the 54 patients operated on, 47 had a nodule identified on imaging, and 43 showed an adenoma on

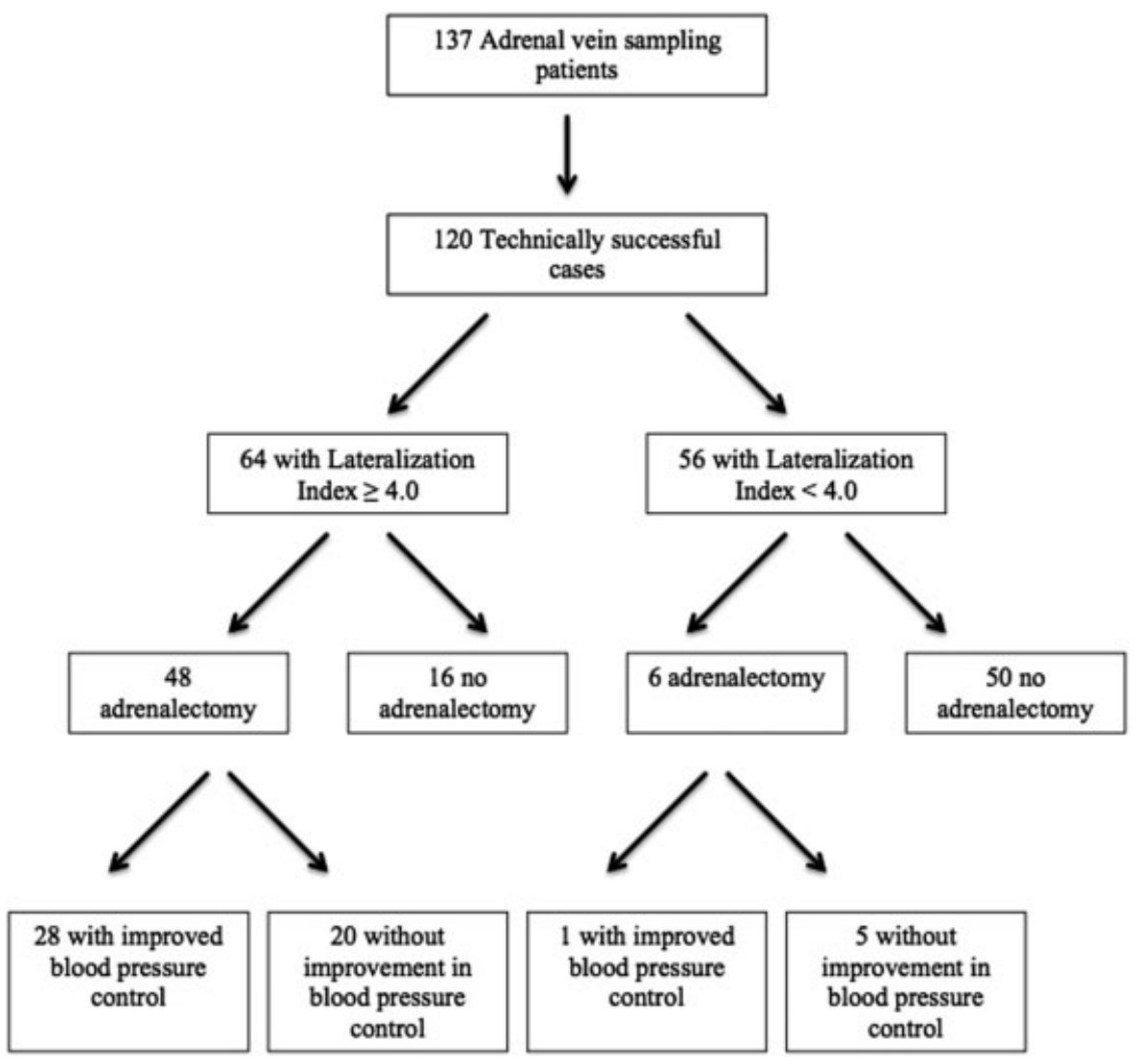

Fig. 1 Flowchart of adrenal vein sampling patients with clinical outcome of blood pressure control. 
Table 1 Summary of adrenalectomy patient characteristics $(n=54)$

\begin{tabular}{|c|c|}
\hline Age, y & $52 \pm 9.9$ \\
\hline Male & $39(72 \%)$ \\
\hline Female & $15(28 \%)$ \\
\hline Caucasian & $42(78 \%)$ \\
\hline African American & $7(13 \%)$ \\
\hline Hispanic & $5(9 \%)$ \\
\hline $\begin{array}{l}\text { No. of antihypertensive medications before } \\
\text { adrenalectomy }\end{array}$ & $3.4 \pm 1.3$ \\
\hline No. of antihypertensives after adrenalectomy & $2.6 \pm 1.4$ \\
\hline eGFR prior to AVS & $86 \pm 21$ \\
\hline eGFR most recent postadrenalectomy & $78 \pm 28$ \\
\hline \multicolumn{2}{|l|}{ Surgical pathology } \\
\hline Adenoma & $43(80 \%)$ \\
\hline Hyperplasia & $11(20 \%)$ \\
\hline
\end{tabular}

Abbreviations: AVS, adrenal vein sampling; eGFR, estimated glomerular filtration rate.

Note: Mean \pm standard deviation.

surgical pathology, while the remaining showed hyperplasia. This discrepancy may in part be due to the nodular component of the gland being macerated upon laparoscopic surgical excision as many of the nodules were subcentimeter. The presence of a nodule on preoperative imaging was associated with clinical improvement after adrenalectomy $(p=0.022)$ with 29 patients achieving improved blood pressure control.

The patients who underwent adrenalectomy were further stratified into groups based on the number of antihypertensives they were taking prior to AVS to determine if there is an association between medication usage prior to surgical treatment and clinical outcomes after surgery. The mean number of antihypertensive medications was $3.4 \pm 1.3$ prior to adrenalectomy and $2.6 \pm 1.4$ after. Patients taking five or more antihypertensives had a statistically significant association with successful clinical outcomes postadrenalectomy $(p=0.007)$. Nine patients in our population were taking five or more antihypertensive medications prior to AVS, all of which showed clinical improvement after adrenalectomy.

In patients undergoing adrenalectomy, the mean eGFR was $86 \pm 21 \mathrm{~mL} / \mathrm{min} / 1.73 \mathrm{~m}^{2}$ prior to AVS and $78 \pm 28 \mathrm{~mL} / \mathrm{min} /$

Table 2 Clinical outcomes associated with surgical pathology

\begin{tabular}{|c|l|l|}
\hline Treatment successful & $\mathbf{L I} \geq \mathbf{4 . 0}$ & $\mathbf{L I}<\mathbf{4 . 0}$ \\
\hline Adenoma & 25 & 0 \\
\hline Hyperplasia & 3 & 1 \\
\hline Treatment failed & $\mathrm{LI} \geq \mathbf{4 . 0}$ & $\mathbf{L I}<\mathbf{4 . 0}$ \\
\hline Adenoma & 16 & 2 \\
\hline Hyperplasia & 4 & 3 \\
\hline
\end{tabular}

Abbreviation: LI, lateralization index.
$1.73 \mathrm{~m}^{2}$ after adrenalectomy. The mean decline in eGFR from baseline to follow-up after adrenalectomy for those that showed clinical improvement was $7.5 \%$ compared with $15.8 \%$ in those who did not meet criteria to undergo adrenalectomy. End-organ damage to the kidney was defined as a decline in eGFR greater than or equal to $30 \%$ from baseline to follow-up postadrenalectomy as a secondary measure of treatment failure. In this analysis, both reduction of antihypertensive medications and preserved renal function were used in combination as outcomes to indicate successful treatment. Thus, improved blood pressure control and stability of eGFR were both required to consider the surgical treatment a success. There were 43 patients with $\mathrm{LI}>4.0$ who underwent adrenalectomy and had data of pre-AVS and postoperative measurement of renal function available. Twenty-two (51\%) of these patients had treatment success by both outcomes, while 21 patients (49\%) failed treatment by one or both outcomes. Of particular note, 11 of these 21 patients demonstrated preserved renal function despite persistent hypertension. The mean decline in eGFR for these 11 patients was $1.6 \%$, while the mean decline in eGFR for the 22 patients with treatment success by both outcomes was $1.4 \%$. Fisher's exact test revealed that treatment success by both measures together was associated with the identification of a discrete nodule on preoperative imaging $(p=0.048)$.

Although the number of patients was small (see - Table 1), it was noted that Hispanic patients had a lower rate of clinical improvement after adrenalectomy with none of the five surgically treated Hispanic patients achieving success by both outcomes despite all five having a nodule on imaging. There were seven African American patients in the study group who underwent adrenalectomy and four achieved clinical success by both outcomes all of which had a nodule on imaging. Caucasians represented the majority of the patients undergoing adrenalectomy of which there were 42 . Twenty-one achieved clinical success by both outcomes, 17 of whom had a nodule on imaging. In addition, 9 out of the 15 female patients who underwent adrenalectomy were successfully treated when both outcomes were considered, all of which had a nodule seen on imaging.

\section{Discussion}

This retrospective review of all AVS cases performed at our institution over a 5-year period was undertaken to determine the preoperative factors among patients with PA that were associated with successful control of hypertension. It has been shown that AVS significantly improves selection of patients likely to benefit from adrenalectomy. ${ }^{12-14}$ However, these studies have focused primarily on the ability of AVS to identify the cause of PA rather than to predict patients who would have a successful clinical result based on the combination of imaging findings and AVS measures. Another study has suggested that obesity, elevated creatinine, left ventricular hypertrophy, and male gender are associated with treatment failure after adrenalectomy. ${ }^{15}$ Our data show identification of a discrete adrenal nodule on preoperative 
imaging to be associated with treatment success in patients who underwent adrenalectomy after AVS. Further, in our population, patients who did not have an adrenal nodule on preoperative imaging were less likely to have improvement in blood pressure control after adrenalectomy.

Previous studies have identified those patients on fewer antihypertensives as more likely to achieve complete cure. ${ }^{9,16}$ Our findings are consistent with these studies, as all three patients who achieved complete cure were on less than three antihypertensives prior to adrenalectomy. In contrast to this, however, we show that those patients on five or more antihypertensives prior to AVS actually had a higher rate of treatment success measured by decrease in the number of antihypertensives alone than those on fewer medications. This could be reflective of a high prevalence of concomitant essential hypertension in our population with the decrease in medication usage due to treatment of the component produced by the adrenal pathology. Alternatively, nonadherence to prescribed medication regimens could explain this finding; however, this is difficult to determine in a retrospective analysis. Although these patients did not achieve a complete cure, the improvement in hypertension from adrenalectomy even in the most drugresistant patients may improve cardiovascular health.

The importance of controlling hypertension is in reducing morbidity and mortality related to end-organ damage. Reports in the literature suggest that a decline in eGFR more than $30 \%$ over 2 years is associated with increased risk of ESRD and mortality. ${ }^{11}$ A less rapid decline in renal function is seen in those patients undergoing adrenalectomy for PA. In our study, patients who underwent successful adrenalectomy had an average decline in eGFR of $7.5 \%$ over an average 8.4-month time interval compared with $15.8 \%$ in those who did not meet criteria for adrenalectomy and were treated only medically. This finding is consistent with prior reports that patients with PA have a more rapid rate of decline in GFR than those with essential hypertension. ${ }^{4,17}$ In part, this may be due to the hypertensive effects with resultant glomerulosclerosis and arteriolosclerosis ${ }^{18}$; however, reports have shown that microalbuminuria associated with PA may also be responsible for the deleterious effects on renal function. ${ }^{19-22}$ This possibly accounts for the 11 patients in our population with stable renal function after adrenalectomy despite having persistent hypertension. This is further supported by the average decline in eGFR of these patients of only $1.6 \%$, which is similar to the $1.4 \%$ decline seen in those patients with both improvement of hypertension and relative preservation of renal function. Nine of these 11 patients also showed a discrete nodule on imaging.

To our knowledge, few studies looked into factors associated with clinical success that combined both improved blood pressure control and renal function as outcomes in the surgical treatment of PA. With both outcomes considered, we show that the identification of a discrete nodule on preoperative imaging may be of importance in predicting clinical success. Prior studies that have created normograms for predicting patients who are likely to be cured by adrenalectomy have identified factors such as age, gender, body mass index, duration of hypertension, and number of antihypertensive drugs to be associated with clinical outcomes. ${ }^{23-25}$ However, these studies have not evaluated the importance of imaging in guiding treatment. Our findings suggest that careful attention should be paid to imaging findings prior to performing adrenalectomy in the absence of a discrete adrenal nodule.

Although a prior study has shown that similar rates of resistant hypertension are due to PA in both Caucasians and African Americans, ${ }^{26}$ there have been no studies demonstrating the rates of treatment success after adrenalectomy in each of these subgroups. We investigated clinical outcomes in Hispanic, African American, and Caucasian subgroups. In our population, Hispanic patients tended to have poorer outcomes after adrenalectomy than African Americans and Caucasians. The difference in rates of treatment success may indicate that genetic variability plays a role in the curability of PA.

Prior studies have shown that the presence of an adenoma at surgical pathology predicted successful clinical outcome. ${ }^{9,27}$ In our study, however, this distinction was less clear. While patients with a nodule on imaging were more likely to have an adenoma identified on surgical pathology, only $61 \%$ of adenomas resected resulted in a favorable effect on blood pressure control. One possible explanation is the coexistence of essential hypertension in patients without improvement after surgery. Alternatively, these patients may have had coexistent adrenal hyperplasia in the contralateral gland which was unmasked after resection of the hypersecreting nodule.

There are several limitations of our study that we recognize. Although all CT and MR images that were available for review were evaluated by a board-certified radiologist at our institution and deemed of adequate quality for interpretation, not all studies had images directly available for review by the authors, and therefore, the quality of these studies could not be confirmed. Therefore, in some cases, there was reliance on interpretations provided in the radiology report constructed by radiologists outside of our institution. Further, this study was a retrospective review of patient charts and all data points were not available for every individual, thus decreasing the number of patients who could be included in each analysis. In particular, reliable estimates of mean blood pressure before and after adrenalectomy were not available for every patient in the study group; therefore, this measure was not included as a primary outcome of clinical success, instead surrogate measures of antihypertensive medication burden and end-organ damage to the kidneys were used. Although the timeframe of the present study averaged 1.1 years when all AVS patients were considered, those who underwent adrenalectomy only spanned an average of 8.4 months from pre-AVS to postadrenalectomy follow-up. Thus, our outcome data are limited to this relatively short follow-up interval and conclusions about long-term success rates may not necessarily be the same. Our cohort consisted predominately of Caucasians with African Americans and Hispanics less represented. Thus, the small numbers of patients in the latter two ethnic groups may limit generalizability. 
In conclusion, we have shown that improvement in blood pressure control and preserved renal function after adrenalectomy in patients with PA is associated with the identification of an adrenal nodule on preoperative imaging. In our cohort, severity of drug-resistant hypertension and ethnic differences in outcomes were also seen. Our findings support that preoperative imaging findings should be carefully considered in determining which patients should undergo surgery for PA.

\section{Conflict of Interest}

None of the authors report any relevant conflict of interest. The Institutional Review Board of our institution approved the study.

\section{References}

1 Melby JC, Spark RF, Dale SL, Egdahl RH, Kahn PC. Diagnosis and localization of aldosterone-producing adenomas by adrenal-vein catheterization. N Engl J Med 1967;277(20):1050-1056

2 Mulatero P, Monticone S, Bertello C, et al. Long-term cardio- and cerebrovascular events in patients with primary aldosteronism. J Clin Endocrinol Metab 2013;98(12):4826-4833

3 Savard S, Amar L, Plouin PF, Steichen O. Cardiovascular complications associated with primary aldosteronism: a controlled crosssectional study. Hypertension 2013;62(02):331-336

4 Sechi LA, Novello M, Lapenna R, et al. Long-term renal outcomes in patients with primary aldosteronism. JAMA 2006;295(22): 2638-2645

5 Reincke M, Rump LC, Quinkler M, et al; Participants of German Conn's Registry. Risk factors associated with a low glomerular filtration rate in primary aldosteronism. J Clin Endocrinol Metab 2009;94(03):869-875

6 Burshteyn M, Cohen DL, Fraker DL, Trerotola SO. Adrenal venous sampling for primary hyperaldosteronism in patients with concurrent chronic kidney disease. J Vasc Interv Radiol 2013;24(05): 726-733

7 Lumachi F, Ermani M, Basso SM, Armanini D, Iacobone M, Favia G. Long-term results of adrenalectomy in patients with aldosteroneproducing adenomas: multivariate analysis of factors affecting unresolved hypertension and review of the literature. Am Surg 2005;71(10):864-869

8 Rossi GP, Bolognesi M, Rizzoni D, et al. Vascular remodeling and duration of hypertension predict outcome of adrenalectomy in primary aldosteronism patients. Hypertension 2008;51(05): 1366-1371

9 Wang W, Hu W, Zhang X, Wang B, Bin C, Huang H. Predictors of successful outcome after adrenalectomy for primary aldosteronism. Int Surg 2012;97(02):104-111

10 Pirvu A, Naem N, Baguet JP, Thony F, Chabre O, Chaffanjon P. Is adrenal venous sampling mandatory before surgical decision in case of primary hyperaldosteronism? World J Surg 2014;38(07): 1749-1754

11 Coresh J, Turin TC, Matsushita K, et al; CKD Prognosis Consortium. Decline in estimated glomerular filtration rate and subsequent risk of end-stage renal disease and mortality. JAMA 2014; 311(24):2518-2531

12 Nwariaku FE, Miller BS, Auchus R, et al. Primary hyperaldosteronism: effect of adrenal vein sampling on surgical outcome. Arch Surg 2006;141(05):497-502, discussion 502-503

13 Young WF, Stanson AW, Thompson GB, Grant CS, Farley DR, van Heerden JA. Role for adrenal venous sampling in primary aldosteronism. Surgery 2004;136(06):1227-1235

14 Rossi GP, Sacchetto A, Chiesura-Corona M, et al. Identification of the etiology of primary aldosteronism with adrenal vein sampling in patients with equivocal computed tomography and magnetic resonance findings: results in 104 consecutive cases. J Clin Endocrinol Metab 2001;86(03):1083-1090

15 Graham UM, Ellis PK, Hunter SJ, Leslie H, Mullan KR, Atkinson AB. 100 cases of primary aldosteronism: careful choice of patients for surgery using adrenal venous sampling and CT imaging results in excellent blood pressure and potassium outcomes. Clin Endocrinol (Oxf) 2012;76(01):26-32

16 Waldmann J, Maurer L, Holler J, et al. Outcome of surgery for primary hyperaldosteronism. World J Surg 2011;35(11): $2422-2427$

17 Wu VC, Kuo CC, Wang SM, et al; TAIPAI Study Group. Primary aldosteronism: changes in cystatin C-based kidney filtration, proteinuria, and renal duplex indices with treatment. J Hypertens 2011;29(09):1778-1786

18 Horita $\mathrm{Y}$, Inenaga $\mathrm{T}$, Nakahama $\mathrm{H}$, et al. Cause of residual hypertension after adrenalectomy in patients with primary aldosteronism. Am J Kidney Dis 2001;37(05):884-889

19 Fourkiotis V, Vonend O, Diederich S, et al; Mephisto Study Group. Effectiveness of eplerenone or spironolactone treatment in preserving renal function in primary aldosteronism. Eur J Endocrinol 2012;168(01):75-81

20 Iwakura Y, Morimoto R, Kudo M, et al. Predictors of decreasing glomerular filtration rate and prevalence of chronic kidney disease after treatment of primary aldosteronism: renal outcome of 213 cases. J Clin Endocrinol Metab 2014;99(05):1593-1598

21 Turchi F, Ronconi V, di Tizio V, Ceccoli L, Boscaro M, Giacchetti G. Primary aldosteronism and essential hypertension: assessment of cardiovascular risk at diagnosis and after treatment. Nutr Metab Cardiovasc Dis 2014;24(05):476-482

22 Rossi GP, Sechi LA, Giacchetti G, Ronconi V, Strazzullo P, Funder JW. Primary aldosteronism: cardiovascular, renal and metabolic implications. Trends Endocrinol Metab 2008;19(03):88-90

23 Wachtel H, Cerullo I, Bartlett EK, et al. Long-term blood pressure control in patients undergoing adrenalectomy for primary hyperaldosteronism. Surgery 2014;156(06):1394-1402, n1402-n1403

24 Utsumi T, Kamiya N, Endo T, et al. Development of a novel nomogram to predict hypertension cure after laparoscopic adrenalectomy in patients with primary aldosteronism. World J Surg 2014;38(10):2640-2644

25 Zarnegar R, Young WF Jr, Lee J, et al. The aldosteronoma resolution score: predicting complete resolution of hypertension after adrenalectomy for aldosteronoma. Ann Surg 2008;247(03):511-518

26 Calhoun DA, Nishizaka MK, Zaman MA, Thakkar RB, Weissmann P. Hyperaldosteronism among black and white subjects with resistant hypertension. Hypertension 2002;40(06):892-896

27 Celen O, O’Brien MJ, Melby JC, Beazley RM. Factors influencing outcome of surgery for primary aldosteronism. Arch Surg 1996; 131(06):646-650 